\title{
Effects of Excluded Volume and Hydrodynamic Interactions on the Behavior of Isolated Bead-Rod Polymer Chains in Shearing Flow
}

\author{
Indranil Saha Dalal \\ Dept. of Chemical Engineering, University of Michigan, Ann Arbor, MI \\ Chih-Chen Hsieh \\ Dept. of Chemical Engineering, National Taiwan University, Taipei, 10617 Taiwan \\ Alex Albaugh and Ronald G. Larson \\ Dept. of Chemical Engineering, University of Michigan, Ann Arbor, MI 48109 \\ DOI 10.1002/aic.14317 \\ Published online January 13, 2014 in Wiley Online Library (wileyonlinelibrary.com)
}

\begin{abstract}
A detailed study of the effects of hydrodynamic interaction (HI) and excluded volume (EV), on isolated bead-rod chains in shear flows, where the "rods" are mimicked by stiff Fraenkel springs is presented. It is observed that the deformation behavior at weak and intermediate shear rates is qualitatively similar to that observed for polymer chains in the absence of $E V$ and HI, while that at high-shear rates is sensitive to modeling details and chain resolution. Our simulations with varying degrees of resolution reveal universality in chain behavior in the presence of EV (without HI), while the onset of the transition to a compressed chain in the presence of HI (without EV) shifts to higher shear rates with increasing chain resolution. The results also highlight the success of the bead-spring models in predicting the chain behavior in shear flows in the presence of HI, when the HI parameter is appropriately chosen. (C) 2014 American Institute of Chemical Engineers AIChE J, 60: 1400-1412, 2014
\end{abstract}

Keywords: complex fluids, simulation, multiscale, rheology, polymer properties

\section{Introduction}

The deformation of polymer chains subjected to shear and other flows has attracted increasing interest, especially since Bird and coworkers published their textbook on molecular modeling of polymer rheology. ${ }^{1}$ This textbook clearly defined the bead-spring and bead-rod models for polymer molecules and provided solutions for their responses in flow fields for various cases, including the effects of hydrodynamic interactions and excluded volume. The clear explanations and thorough coverage has made this work a standard reference source for all workers in the field, and has inspired much of what has transpired in the field, including this article.

In recent years, the tools of single-molecule imaging experiments $^{2-4}$ and Brownian dynamics simulations of beadspring and bead-rod chains ${ }^{5-8,11,12}$ have allowed the problems discussed in Bird et al. to be explored in much greater detail. These recent studies show that the time-averaged polymer stretch in the flow direction increases with shear rate up to a high-shear-rate plateau that is about $40 \%$ of the fully extended chain length. ${ }^{7}$ The plateau occurs at less than full extension because the chain undergoes incessant end-

\footnotetext{
Correspondence concerning this article should be addressed to R. G. Larson at rlarson@umich.edu.
}

(C) 2014 American Institute of Chemical Engineers over-end tumbling, which varies the end-to-end distance from zero to full extension, and produces an average of around $40 \%$ of full extension on the plateau. This plateau was believed to be the asymptotic response of the chain at high shear rates, until some surprising results were observed in a bead-rod simulation study by Sendner and Netz, ${ }^{9}$ who observed that the chain size, measured by the radius of gyration, decreases at extremely high shear rates, above the rates at which the plateau is observed. Results from the same study also indicated that this chain shrinkage was hindered by excluded volume interactions between beads, but was amplified by hydrodynamic interactions (HI) between them. Using BD simulations of long bead-spring and bead-rod chains in the absence of $\mathrm{HI}$ and excluded volume (EV), in Refs. 11 and 12, three distinct shear-rate regimes were observed. Regime 1 spans weak to moderate shear rates where the chain elongation increases with shear rate. Regime 2 covers intermediate rates where the chain stretch shows a plateau. However, Regime 3 encompasses extremely highshear rates where a decrease in chain elongation is observed. The behaviors in the first two regimes are insensitive to refinement of the chain, but the third regime is suppressed in the absence of $\mathrm{HI}$ and $\mathrm{EV}$ if rods with bending potential are introduced to increase the refinement of the polymer model. The anomalous chain shrinkage in Regime 3 with insufficient chain refinement occurs due to sequential flipping of individual rods, which slows the process of chain stretching 
to the point that end-over-end tumbling events occur before the chain becomes completely extended. If each rod is replaced by multiple shorter rods with a bending potential to produce a chain with the same overall chain length and coil size, however, full extension is nearly attained before a tumbling event, and the decrease in elongation is avoided, thereby extending Regime 2 and avoiding the onset of Regime 3. However, HI and EV need to be included in these simulations to fully resolve the deformation behavior of realistic chains in the presence of shear.

In this article, we therefore investigate chain deformation in shear when EV interactions and $\mathrm{HI}$ are acting between the beads, thus completing the study of chain deformation due to shearing flows presented earlier. ${ }^{11,12} \mathrm{We}$ add bead-bead excluded volume interactions and HI separately and observe the effects of each on chain elongation (in the flow direction), and chain thickness (in the shear-gradient direction), relative to those obtained earlier without either of these additional mechanisms. Although some indications of the elongation behavior with $\mathrm{HI}$ and EV were given earlier in Ref. 9, the details and the relevance of those observations to actual systems are not fully clear, since only a single model (the bead-rod model) was used for a single-chain length (100 rods). In this article, after explaining our methods in the section "Simulation methods", we present and discuss our observations in the section "Results and discussion". Our results with $\mathrm{HI}$ and $\mathrm{EV}$ for the chain stretch and width can again be categorized using three distinct regimes, but the trends at high shear rates change depending on which mechanism is acting between the beads. In what follows, we show that rather weak EV interactions suffice to suppress the chain shrinkage in Regime 3, while increasing HI systematically increases the chain shrinkage in Regime 3, producing a highly coiled state at high-shear rates in the absence of any excluded volume interactions. (Note that the high-shear rates in Regime 3 are not necessarily experimentally accessible unless the solvent is made very viscous, and/or the chain is quite stiff, since for flexible chains the highest shear rate is likely limited by experimental limits on shear rate, or by chain scission, as are discussed in Ref. 11.) Additionally, our results with chains at varying degrees of resolution indicate universality in chain deformation behavior in the presence of EV interactions. We will also show that the sharp decrease in coil stretch in the presence of HI (without EV) shown in Ref. 9 is pushed to arbitrarily high shear rates with increasing levels of resolution, for semiflexible rodlike chains. The simulations with the CG model highlight its ability to predict the conformational properties of the chain, when the hydrodynamic interaction parameter is fixed appropriately.

A brief note needs to be added here regarding the terminology for excluded volume interactions used in this article. The designation "EV" will denote chains in which beads interact only through short-range repulsive interactions. It will be shown later in this article that this approach reproduces the well-known scaling laws for a chain in a good solvent. However, in a real chain, the interaction between atoms is repulsive at short range and can be attractive at larger distances. In the finely resolved chains used in this article (resolved to length scales of the Kuhn length or shorter), we can also include relatively long-range attractive interactions, in addition to the short-range repulsive interactions. The case in which the short-range repulsion and longrange attraction are tuned such that the chain size (measured by the radius of gyration) matches that of a theta coil (i.e., a random walk, without any excluded volume interactions) will be referred to as "EV-theta", where the long-range attraction is just large enough to nullify the effect of repulsion at short-range. In this article, the repulsive and attractive interactions are modeled using the Lennard-Jones potential, which is typically used between atoms in molecular dynamics simulations. Note that we only consider the cases of "EV" and "EV-theta" in this article. Cases in which stronger attractive interactions lead to coil collapse will be considered in future work.

\section{Simulation Methods}

The model of the polymer chain, symbols for variables, and the governing equations used in this article are the same as discussed in Ref. 11, unless otherwise mentioned. Briefly, using a characteristic length scale of one rod in a polymer chain model $\left(l_{\text {rod }}\right)$, consisting of beads connected by stiff springs that mimic inextensible rods, and a time scale of $\zeta l_{\text {rod }}^{2} / k_{B} T$, where $\xi$ is the bead drag coefficient, we arrive at the following equation of motion without $\mathrm{HI}$ and $\mathrm{EV}$

$$
\frac{d \vec{r}_{i}^{*}}{d t^{*}}=\kappa^{*} \cdot \vec{r}_{i}^{*}+\left[\vec{F}_{i+1}^{S *}-\vec{F}_{i}^{S *}\right]+\sqrt{\frac{6}{\Delta t^{*}}} \vec{n}_{i}
$$

where all the variables have the same significance as described in Ref. 11 and the corresponding dimensionless variables are denoted by a "*" in the superscript. For this study, we use three different types of chain models to understand the numerical artifacts related to the level of resolution: (1) the coarse-grained bead-spring model where each spring represents a total of $v$ Kuhn steps (denoted by the "CG" model). For this study, the Cohen-Padé approximation is used as the spring force law (details provided in Ref. 11), (2) the "bead-rod" (or BR) model used earlier in Refs. 11 and 12 where each "rod" is mimicked by a stiff Fraenkel spring and represents one Kuhn length of the polymer chain $b_{K}$; thus, for the BR model $l_{\text {rod }}=b_{K}$ where $l_{\text {rod }}$ is the rod length, and (3) the "fine-grained" (FG) model which also consists of beads connected by "rods" (again mimicked by stiff Fraenkel springs), where a harmonic bending potential acts between consecutive rods in the chain (details given in Ref. 12). The bending stiffness parameter can be tuned to obtain a desired level of resolution, i.e. a desired number of rods per Kuhn step. Thus, for the FG model, $l_{\text {rod }}<b_{K}$. Further details and values of the bending stiffness required to obtain different levels of discretization are provided in Ref. 12. Most of the results in this study use the BR model, and FG models are used to highlight the importance of the inclusion of finer details in the results. Simulations with the CG model, in the presence of HI, will also be carried out to test their ability to capture the behavior of finer-grained models in the predictions of chain stretch at different shear rates. In both the BR and FG models, we mimic inextensible "rods" with Fraenkel springs with a force law given as

$$
\vec{F}=K(|\vec{Q}|-1) \frac{\vec{Q}}{|\vec{Q}|}
$$

where $K$ and $\vec{Q}$ are the stiffness constant of the Fraenkel spring and the spring end-to-end vector, respectively. With this spring force expression, Eq. 1 becomes 


$$
\begin{gathered}
\frac{d \vec{r}_{i}^{*}}{d t^{*}}=\kappa \cdot \vec{r}_{i}^{*}+K^{*}\left[\left\{\left(\left|\vec{Q}_{i+1}^{*}\right|-1\right) \frac{\vec{Q}_{i+1}^{*}}{\left|\vec{Q}_{i+1}^{*}\right|}\right\}-\left\{\left(\left|\vec{Q}_{i}^{*}\right|-1\right) \frac{\vec{Q}_{i}^{*}}{\left|\vec{Q}_{i}^{*}\right|}\right\}\right] \\
+\sqrt{\frac{6}{\Delta t^{*}}} \vec{n}_{i}
\end{gathered}
$$

where the value of the parameter $K^{*}=\frac{K l_{\text {rod }}^{2}}{k_{B} T}$ can be tuned to adjust the stiffness of the Fraenkel springs in the polymer chain. Following the approach in Ref. 11, a value of $K^{*}=10^{4}$ is used for $\dot{\gamma}<1$ and $K^{*}=10,000 \dot{\gamma}$ for $\dot{\gamma} \geq 1$ to prevent overstretching of the springs, unless otherwise mentioned. For the FG model, an additional force due to the bending potential between consecutive rods will also be present.

To investigate the effects of excluded volume interactions in either the BR or the FG models, the following LennardJones potential (LJ) is introduced for the nonbonded interactions between beads (similar to the approach in Ref. 9)

$$
U_{i j}=4 \varepsilon\left[\left(\frac{\sigma}{\left|\vec{r}_{i j}\right|}\right)^{12}-\left(\frac{\sigma}{\left|\vec{r}_{i j}\right|}\right)^{6}\right]
$$

where $\left|\vec{r}_{i j}\right|=\left|\vec{r}_{i}-\vec{r}_{j}\right|$ represents the inter-bead distance, $\sigma$ represents the "diameter" of the beads and $\varepsilon$ is the interaction strength. For the BR model, the excluded volume interactions are only computed between beads that are separated by 2 or more rods, i.e., beads that are not consecutive to each other in the chain. In the FG model, the LJ interactions are excluded for all bonded neighbors within a Kuhn step (i.e., for an FG chain where four rods constitute one Kuhn step, the LJ interactions are excluded between beads that are separated by four rods or less), so that the scheme is consistent with that used for the BR model, where the excluded volume interactions are excluded between bonded neighbors (i.e., within one Kuhn step, since every rod in the BR model represents a Kuhn step). If the force due to the excluded volume interactions is included, the equation for the evolution of bead trajectories for the BR model is given as

$$
\begin{aligned}
& \frac{d \vec{r}_{i}^{*}}{d t^{*}}=\kappa \cdot \vec{r}_{i}^{*}+K^{*}\left[\left\{\left(\left|\vec{Q}_{i+1}^{*}\right|-1\right) \frac{\vec{Q}_{i+1}^{*}}{\left|\vec{Q}_{i+1}^{*}\right|}\right\}-\left\{\left(\left|\vec{Q}_{i}^{*}\right|-1\right) \frac{\vec{Q}_{i}^{*}}{\left|\vec{Q}_{i}^{*}\right|}\right\}\right] \\
& +4 \varepsilon^{*} \sum_{j=0,|i-j| \geq 2}^{N} \frac{1}{\left|\vec{r}_{i j}^{*}\right|}\left[12\left(\frac{\sigma^{*}}{\left|\vec{r}_{i j}^{*}\right|}\right)^{12}-6\left(\frac{\sigma^{*}}{\left|\vec{r}_{i j}{ }^{*}\right|}\right)^{6}\right] \frac{\vec{r}_{i j}^{*}}{\left|\vec{r}_{i j}{ }^{*}\right|}+\sqrt{\frac{6}{\Delta t^{*}}} \vec{n}_{i}
\end{aligned}
$$

where the dimensionless parameters $\varepsilon^{*}=\frac{\varepsilon}{k_{B} T}$ and $\sigma^{*}=\frac{\sigma}{b_{K}}$ are used to adjust the interaction strength and the bead diameter for the Lennard-Jones interactions, respectively (Note that, for the BR model, we have $l_{\text {rod }}=b_{K}$ ). Also, for the FG model, there will be an additional force due to the harmonic bending potential between consecutive rods and the excluded volume interactions will only be computed between beads that are separated by a distance larger than one Kuhn step along the chain contour, as mentioned earlier. Also as mentioned earlier, in the BR model, the excluded volume interactions between beads are modeled in two ways (1) only the repulsive part of the $\mathrm{LJ}$ potential is retained (thus dropping the attractive part of the interaction involving the $\left(\frac{\sigma}{\left|r_{i j}\right|}\right)^{6}$ term), and (2) the full LJ potential is used with the value of $\varepsilon^{*}$ tuned such that the radius of gyration of the chain at equilibrium is the same as that of a theta coil (where both HI and bead-bead excluded volume interactions are absent). The first represents a traditional way of including the excluded volume interactions, which leads to coil expansion, while the second method includes both short-range repulsion and longer-range attraction, such that their effects on equilibrium coil size cancel out. As discussed earlier, the first type of interaction will be denoted "EV", whereas "EV-theta" represents the second type that produces a theta coil at equilibrium (For a chain with 100 rods studied in this article, a value of $\varepsilon^{*}=0.44$ is needed to produce a theta coil, when $\sigma^{*}=1.0$ ). Interaction cut-off radii of $2.5 \sigma^{*}$ and $2 \sigma^{*}$ are used for EV-theta and EV chains, respectively. For the integrations involving excluded volume interactions, unless otherwise mentioned, a timestep size $\Delta t^{*} \leq\left(1 / K^{*}\right)$ is used. However, for cases with relatively smaller bead "diameter", i.e. $\sigma^{*}<0.5$, a smaller timestep of $\Delta t^{*} \leq\left(1 / 10 K^{*}\right)$ is needed to ensure good convergence. The implicit numerical algorithm discussed in Ref. 11 is used for simulations using EV and EV-theta interactions in this study. As described in Ref. 11, for low shear rates $(\dot{\gamma}<1)$, the total run time with EV depends on the number of rods in the chain $N$ (with $N=N_{K}$ for the BR model) and is usually chosen to be about 100 times the longest relaxation time for chains with $\mathrm{N} \leq 200$ and 50 times the longest relaxation time for $\mathrm{N}>200$, for the BR model. Since the computational time per time step scales as $\mathrm{N}^{2}$ with either EV or EV-theta for computing the pairwise LJ interactions, chains longer than $\mathrm{N}=300$ are not considered either with the BR or the FG model. For the BR model, at higher shear rates $(\dot{\gamma} \geq 1)$, simulations are run for a total strain $\dot{\gamma} t$, where $t$ is the simulation time, of at least $10^{5}$ for $\mathrm{N} \leq 200$ and $5 \times 10^{4}$ for longer chains. Unless otherwise mentioned, the initial $20 \%$ of the simulation is used for attaining a steady state, and the final $80 \%$ of the run is used to calculate the average of each quantity.

The hydrodynamic interactions between the beads are modeled by the Rotne-Prager-Yamakawa (RPY) tensor as in earlier studies ${ }^{8,9}$ using the semi-implicit numerical scheme described in Ref. 8, for all the three chain models used in this study. The selection procedure mentioned earlier for the values of the dimensionless spring stiffness parameter and the time-step size for integration works well even when HI is applied. We observed that, to get accurate results for our case of a BR or a FG chain, where beads are connected by stiff springs that mimic rods, the diffusion tensor $\left(D_{i j}\right.$ defined in Ref. 8) has to be updated after every time step, which was not necessary in Ref. 8 , where the beads were connected by coarse-grained springs. The hydrodynamic radius of the beads is controlled by the parameter $h^{*}$ (defined in Ref. 8), for which a maximum value of 0.5 is used in most of our simulations, representing a hydrodynamic bead diameter equal to the length of one rod, where one rod represents either a Kuhn step or a fraction of a Kuhn step in the BR and FG chains, respectively. With the introduction of HI, the computational time per time step scales roughly as $\mathrm{N}^{3}$, which limits the maximum chain lengths for simulations to $\mathrm{N} \leq 150$ for any chain model. With the BR model with HI, for low-shear rates $(\dot{\gamma} \leq 1)$, the total run time is usually chosen to be more than 100 times the longest relaxation time for chains with $\mathrm{N} \leq 100$ and about 50 times the longest relaxation time for larger chains. At higher shear rates $(\dot{\gamma}>1)$, simulations are run for a total strain of at least 5000 for $\mathrm{N} \leq 100$ and 1,000 for larger chains. As in the case with excluded volume interactions, the average of any quantity is calculated using the final $80 \%$ of the simulation trajectory. 
The same is done for cases where both HI and EV (or EVtheta) are present. However, when both HI and EV are present, the equilibrium coil size is larger than that of the theta coil and, consequently, the end-to-end relaxation time is also larger. Hence, for most cases considered here, the total run time at lower shear rates for chains with both $\mathrm{HI}$ and $\mathrm{EV}$ is much lower than 100 times the longest relaxation time, leading to relatively poorer statistics for smaller shear rates.

For all cases, the rotational relaxation time $(\tau)$ of the polymer chain is calculated by fitting the last $70 \%$ of the autocorrelation function of the chain end-to-end vector in the absence of shear. The Weissenberg number (Wi) is defined as the product of the shear rate and the chain rotational relaxation time (Wi $=\tau \dot{\gamma}$ ). The formulas for the radii of gyration $R_{g x}$ and $R_{g y}$ along the flow and flow-gradient directions, respectively, are given in Ref. 11. In this article, the terms "coil elongation" and "coil thickness" will denote $R_{g x}$ and $R_{g y}$, respectively. Note that, for this article, the terms "rotational relaxation time", "longest relaxation time" and "end-to-end relaxation time" have the same meaning, unless otherwise stated.

In the investigations in Ref. 11 with the BR model without $\mathrm{HI}$ and $\mathrm{EV}$, the rod Peclet number $(\mathrm{Pe})$, based on the relaxation time of one single rod, was defined as $\mathrm{Pe}=\mathrm{Wi} / \mathrm{N}_{\mathrm{K}}{ }^{2}$. Here $\mathrm{N}_{\mathrm{K}}$ is the number of Kuhn steps in the chain. Thus, for the $\mathrm{BR}$ model $\mathrm{N}_{\mathrm{K}}=\mathrm{N}$ and $l_{\text {rod }}=b_{K}$. This definition of $\mathrm{Pe}$ is reasonable if the rod reorientation relaxation time is $\tau_{r}=\tau / \mathrm{N}_{\mathrm{K}}{ }^{2}$, which is only true when $\mathrm{EV}$ and $\mathrm{HI}$ are absent. As shown in Ref. 11, this value of $\tau_{r}$ is related to the characteristic time scale of the simulation $\tau_{0}=\zeta b_{K}^{2} / k_{B} T$ by a prefactor of 0.0338 (equal to $1 / 3 \pi^{2}$ ) that is obtained from the Rouse model. Since the same characteristic time scale of $\tau_{0}=\zeta b_{K}^{2} / k_{B} T$ is used for the simulations in this study using the BR model, we can define $\mathrm{Pe}=0.0338 \dot{\gamma}$, thus, using the same definition of the Peclet number as in Ref. 11 throughout for presenting our results from the BR model. The advantage of this definition is that even when HI and $\mathrm{EV}$ are present, the Peclet number will still be roughly equal to the shear rate times the relaxation time of a single Kuhn segment.

In this article, we use the same definition of Regimes 1 and 2 as described in Ref. 11. Regime 1 represents the range of relatively weak shear rates (or Peclet numbers) where the coil thickness stays constant at its equilibrium value, whereas Regime 2 denotes the intermediate shear rates where the coil elongation approximately reaches a saturation value (ca. 0.2 $\mathrm{L}$ in the absence of $\mathrm{HI}$ and $\mathrm{EV}$, where $\mathrm{L}$ is the contour length of the chain). As we will note later in this article, the variation of coil elongation and thickness at ultra-high shear rates (constituting Regime 3 in Ref. 11) is highly sensitive to the details of the chain model. For the BR model in this study, shear rates with $\mathrm{Pe} \geq 1$ are considered to be in Regime 3 for all cases, unless otherwise specified. As we note later in this article, the chain deformation behavior, as a function of the Weissenberg number, is roughly similar for all models in Regime 1 and in the transition to Regime 2. Hence, the values of $\mathrm{Wi}$ for the end of Regime 1 and the onset of Regime 2, given in Ref. 11, also apply to the results presented here.

\section{Results and Discussion Scaling of relaxation times}

To investigate the scaling of the relaxation times, we use the BR model throughout this section. As mentioned earlier, the rotational relaxation time for long chains scales as $\mathrm{N}_{\mathrm{K}}{ }^{2}$ in the absence of $\mathrm{HI}$ and $\mathrm{EV}$ and as $\mathrm{N}_{\mathrm{K}}{ }^{1.5}$ in the presence of HI without excluded volume interactions, according to the Rouse and Zimm models, respectively. Earlier studies have shown that the relaxation time scales as $\mathrm{N}_{\mathrm{K}}^{2.2}$ for swollen coils when EV is included, in the absence of HI. ${ }^{10}$ However, the BR model used here differs from the Rouse and Zimm models in two significant ways (1) we consider a bead-rod polymer chain, where each "rod" in our chain is actually a stiff Fraenkel spring, mimicking a Kuhn step, which resolves the polymer more finely than does a chain of Hookean springs, where each spring represents several Kuhn steps, and (2) we account for the effects of fluctuations of $\mathrm{HI}$ in our BD simulations, whereas preaveraged HI was used in the Zimm theory. Hence, it is worthwhile to compare the scaling of the rotational relaxation times of our chains with those of the Rouse and Zimm analytical theories, as well as to check whether we can reproduce the scaling laws for a polymer in good solvent by adding EV interactions. To the best of our knowledge, no other study has computed the rotational relaxation times from the bead-rod model when $\mathrm{HI}$ or $\mathrm{EV}$ is present.

Figure 1 shows the variation of the relaxation times of chains of different lengths in the presence of only EV (in the absence of $\mathrm{HI}$ ) and only $\mathrm{HI}$ (in the absence of EV), using the BR model. For all cases with EV, the dimensionless energy scale $\varepsilon^{*}$ in the LJ potential (where we are here including only the repulsive part) and the dimensionless excluded volume diameter of the beads $\sigma^{*}$ (in Eq. 5) are both taken to be unity. For the results with HI, the bead hydrodynamic diameter is taken to be equal to the length of

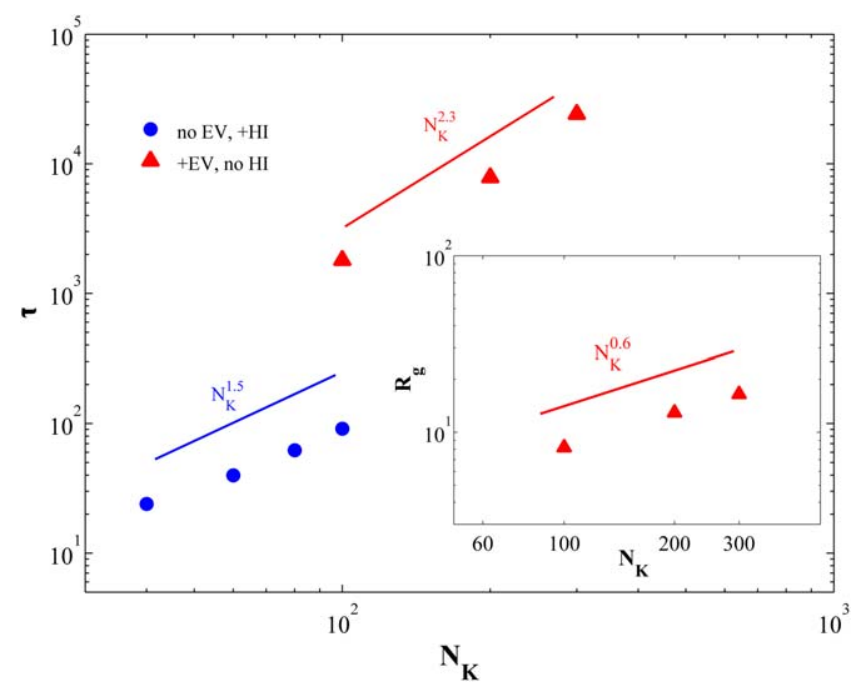

Figure 1. Dependence of rotational relaxation time for the BR model on the number of Kuhn steps, when only excluded volume (EV) or only hydrodynamic interactions $(\mathrm{HI})$ are included.

The "+" sign and "no" indicate the mechanism that is present and not present, respectively. For results with $\mathrm{EV}$, only the repulsive part of the $\mathrm{LJ}$ potential with $\sigma^{*}=1$ is used. The solid lines indicate power laws with exponents of approximately 2.3 and 1.5 for cases with $\mathrm{EV}$ and HI, respectively. The inset shows the variation of the coil radius of gyration at equilibrium with chain length when $\mathrm{EV}$ is active, showing a power law with an exponent of about 0.6. [Color figure can be viewed in the online issue, which is available at wileyonlinelibrary. com.] 


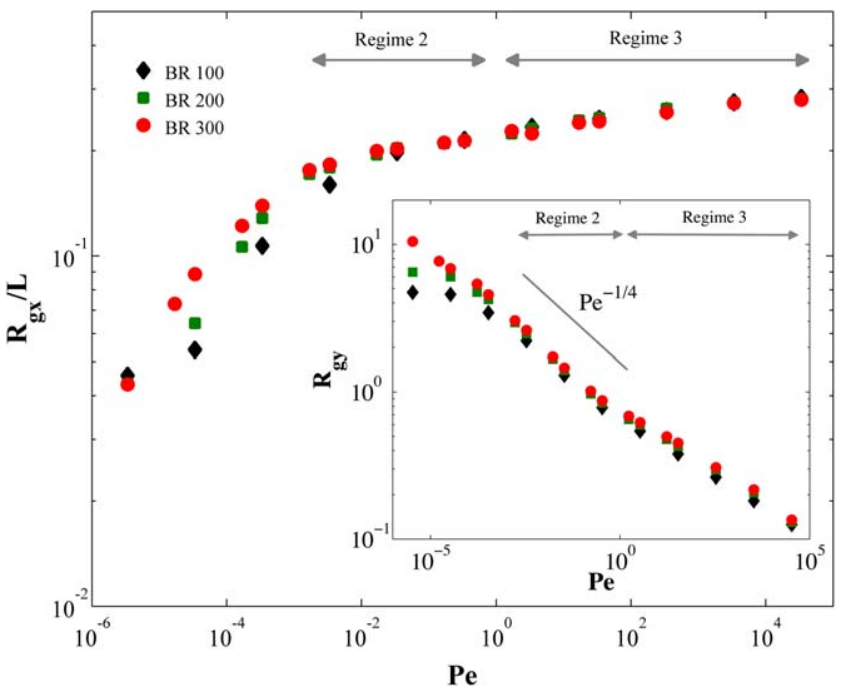

Figure 2. Dependence of the chain elongation normalized by chain contour length $L$ on shear rate for chains of different length, when only EV (and not $\mathrm{HI}$ ) is present.

For all results shown here, EV is modeled by the repulsive part of the $\mathbf{L J}$ potential with $\sigma^{*}=1$. The inset shows the dependence of the coil thickness $R_{g y}$ on shear rate for chains of different length. The solid line marks the region of shear rates where $\boldsymbol{R}_{g y}$ roughly follows a power law with an exponent of about $-1 / 4$, which is the exponent found in Regime 2 in the absence of $\mathrm{HI}$ and EV. ${ }^{11,12}$ The arrows indicate the approximate range of shear rates for Regimes 2 and 3. [Color figure can be viewed in the online issue, which is available at wileyonlinelibrary.com.]

one Kuhn step. We observe that, in the presence of EV and without HI, the exponent for the dependence of relaxation time on chain length is approximately 2.3 (see Figure 1), which is similar to the value of 2.2 reported earlier for polymer chains with $\mathrm{EV} \cdot{ }^{10}$ In the presence of $\mathrm{HI}$ (without $\mathrm{EV}$ ), the exponent is approximately 1.5 , which is consistent with the predictions of the Zimm model. The inset shows the variation of the chain radius of gyration with chain length (in terms of number of Kuhn steps) in the presence of EV (without HI). The exponent of about 0.6 for the power law obtained from our results agrees well with that expected for polymer chains of different lengths in good solvents. Thus, our model used in this article predicts the well-known scaling laws for chains in a good solvent and highlight the generality of the Zimm theory as well.

\section{Effects of EV (without HI)}

The values of the $\mathrm{LJ}$ parameters used here are $\sigma^{*}=1.0$ (i.e., one Kuhn length for the BR model) and $\varepsilon^{*}=1.0$ (i.e., $1 \mathrm{k}_{\mathrm{B}} \mathrm{T}$ ), while accounting for only the repulsive part of the potential. Figure 2 and its inset show the dependence on Peclet number of the average coil elongation and the coil thickness, respectively, for the BR model in shear. The trends obtained for the variation of both $R_{g x}$ and $R_{g y}$ at low to intermediate shear rates, encompassing Regimes 1 and 2, are quite similar to those in the absence of excluded volume interactions (see Refs. 11 and ${ }^{12}$ ), with the "plateau" in $R_{g x}$ that characterizes Regime 2 becoming increasingly evident for longer chains. Also, the value of $R_{g x} / L$ along the plateau is similar to that in the absence of EV. However, interestingly, a true plateau is not

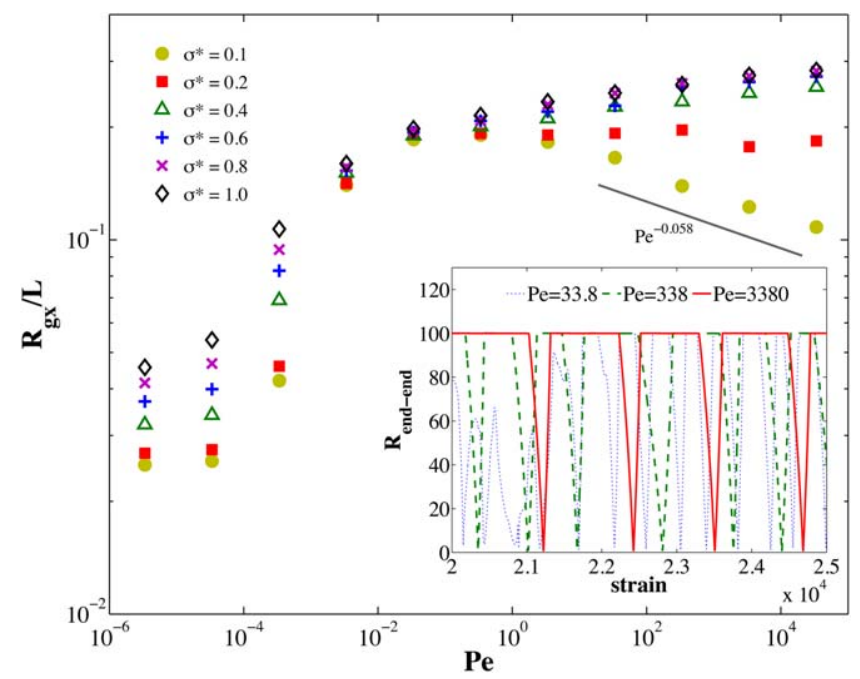

Figure 3. Dependence of chain elongation on Peclet number for varying strength of EV, modeled by different values of $\sigma^{*}$, where EV is modeled by the repulsive part of the LJ potential; the inset shows the time variation (measured in units of strain) of the end-to-end distance of a chain of 100 rods at high shear rates, with $\sigma^{*}=1$ for various $\mathrm{Pe}$.

The strain is the dimensionless time $\dot{\gamma} t$. The inset shows increasing levels of orientational locking as the shear rate increases for $\dot{\gamma}=10^{3}, 10^{4}$ and $10^{5}$, corresponding to $\mathrm{Pe}=33.8,338$ and 3380, respectively. Note that $R_{\text {end-end }}$ is the end-to-end distance of the polymer chain. Hence, for a fully extended chain, $R_{\text {end-end }}$ equals $L$, whereas $R_{g x}$ is about $L / \sqrt{ } 12$. [Color figure can be viewed in the online issue, which is available at wileyonlinelibrary. com.]

obtained, as the coil elongation increases gradually with shear rate instead of reaching a saturation value, as observed earlier for chains without EV and HI. Also note that the decrease in $R_{g x}$ at high-shear rates, observed earlier in Ref. 9 for a chain of 100 rods without EV, is absent for all chain lengths considered. Instead, Regime 3 is characterized by frequent "orientational locking", where occasionally all rods orient in the flow direction, producing a completely stretched chain, which persists for some time, but eventually collapses, only to occur again later aperiodically in time. Similar locked states are observed in bead-rod chains without excluded volume interactions or $\mathrm{HI}$, but only at much higher Peclet numbers, beyond the onset of Regime 3, as noted in Ref. 11. (As discussed in Ref. 11, the locking behavior persists even when the time step is made much smaller, and, thus, does not appear to be an artifact of the numerical scheme used to solve the equations, but represents the behavior of the model at high-shear rates.) Similar to the case of noninteracting beads presented in Ref. $11, R_{g y}$ remains constant at the equilibrium value in Regime 1, and in Regime 2 decreases with a power law exponent of about $-1 / 4$ and is independent of the chain length (inset of Figure 2). In Regime 3, increasing orientational "locking" in the fully extended state causes the average chain dimension in the shear-gradient direction to continue to decrease with shear rate, albeit with a slightly smaller slope than in Regime 2.

This "locking" behavior is shown more clearly in the inset of Figure 3, where the temporal variation of the chain end- 
to-end distance (for a chain of BR 100 rods with $\sigma^{*}=1.0$ ) is presented at different Peclet numbers. A systematic increase in the duration of orientational locking in the fully stretched state is clearly observed with increasing shear rate, which accounts for the slow increase in $R_{g x}$ at high shear rates, in place of a long plateau or decrease in $R_{g x}$ at high-shear rates in Figure 2. As noted earlier in Ref. 11, Regime 3 is characterized by the presence of back-folds where beads come very close to one another, even in the most stretched states that the chain can attain before tumbling. In the presence of excluded volume interactions, back-folds are likely to be hindered by repulsive forces between beads, and so the chain remains locked in the extended state for a considerable duration before tumbling can start.

The mean coil elongation is presented in the main body of Figure 3 , for different values of $\sigma^{*}$ with $\varepsilon^{*}$ fixed at 1.0, indicating that the chain behavior for the BR model in strong shear flows is significantly affected by the bead "diameter" $\left(\sigma^{*}\right)$. A regime in which $R_{g x}$ decreases with increasing shear rate, reminiscent of Regime 3 for ideal bead-rod chains (see Ref. 11), only reappears when $\sigma^{*}$ is reduced to about $10 \%$ of the Kuhn length. Even when $\sigma^{*}$ is reduced to this value, the power-law exponent for the dependence of $R_{g x}$ on shear rate is only around $-0.058( \pm 0.008)$, instead of the value of -0.15 obtained when bead interactions are neglected entirely (shown in Ref. 11). Thus, the coil elongation at high-shear rates for the BR model is extremely sensitive to the strength of excluded volume interactions, even when such interactions are very weak.

A more detailed analysis of the properties of the orientational "locking" behavior is presented in Figure 4. The probability distributions of the end-to-end distance for a BR chain of 100 rods at different Peclet numbers are shown in Figure 4a. A bin size of 5 is used to calculate the distributions, whereas the inset shows the same calculated with a bin size of 0.5 . It is observed that at $\mathrm{Pe}=0.00338(\dot{\gamma}=0.1)$, the distribution is peaked at a low value of coil stretch, which is expected since the polymer chain does not significantly extend at relatively weaker shear rates. The roughly uniform distribution at $\mathrm{Pe}=0.0338$ is reminiscent of those obtained in Ref. 11, for chains without EV at shear rates in Regime 2. However, at higher shear rates, the probability of the fully extended state rises above zero, which indicates a significant proportion of highly stretched states at these shear rates. The probability distributions for a BR chain of 100 rods with no $\mathrm{EV}$, over the same range of shear rates, never shows a such a large proportion of highly stretched states (as shown in Ref. 11).

The probability distributions in the inset of Figure $4 \mathrm{a}$ are calculated from the same trajectories, but for a more refined bin size that is 10 times smaller. These resolve the peak in the distribution in the region of highly stretched states and show the presence of orientational locking as well. Clearly, the locked states occur only at $\mathrm{Pe}=1.69$ or above (i.e., $\mathrm{Pe}>1$ ), where there is a significant probability for the fully extended state. At lower values of Pe, the peak occurs at a highly stretched state, but not a fully stretched one.

Figure $4 \mathrm{~b}$ shows the dependence of the average residence strain $\left(\dot{\gamma} t_{\text {res }}\right)$ in the "locked" state, normalized by the chain length, on shear rate, where $t_{\text {res }}$ denotes the residence time in the "locked" state. Note that here time is made dimensionless with strain rate so that it is consistent with that used in the inset of Figure 3. The residence time is calculated by the duration over which the chain end-to-end distance remains
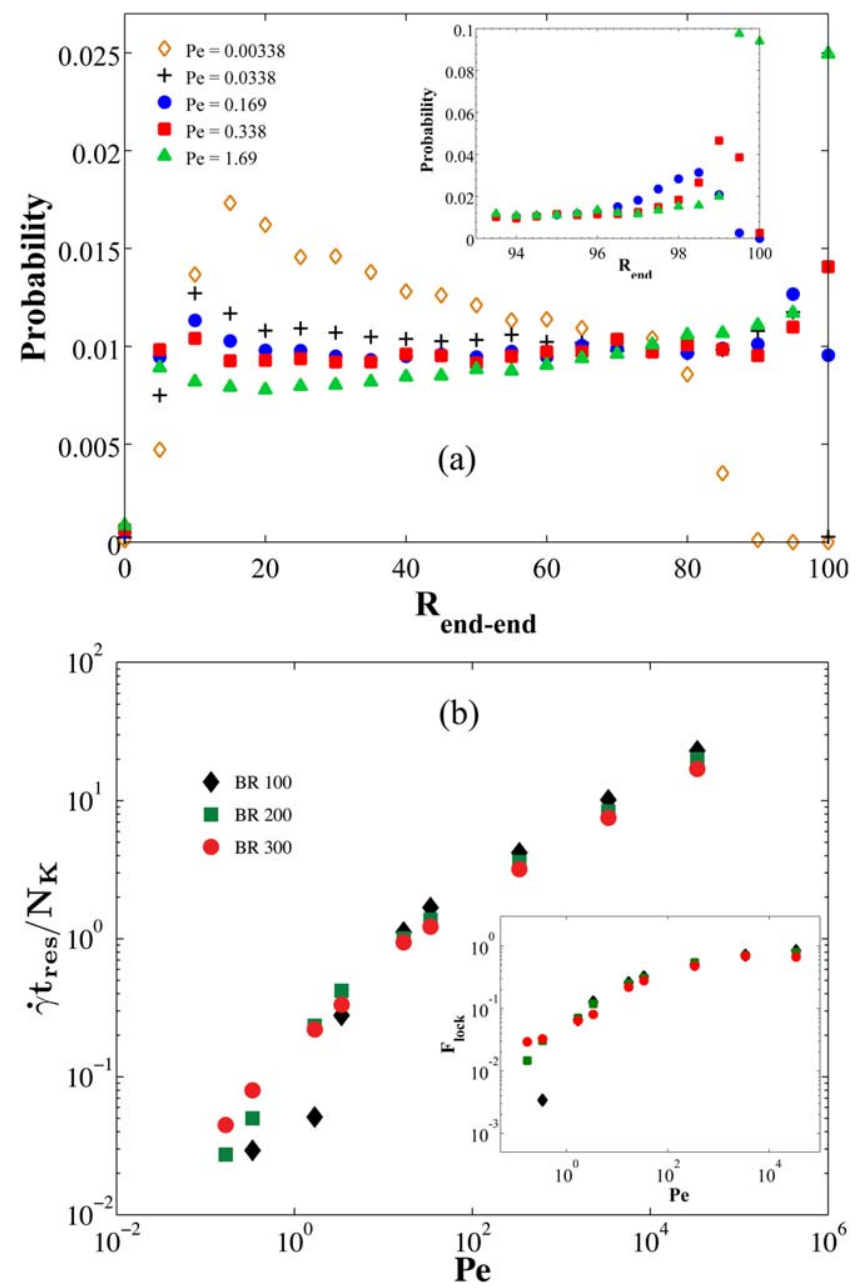

Figure 4. (a) Probability distributions of the chain endto-end distance for a BR chain of 100 rods at different shear rates, expressed as Peclet numbers, calculated using a bin size of 5; the inset shows the same for highly stretched states, using a bin size of 0.5 , and (b) the dependence on Peclet number of the average residence time for a chain in an "orientationally locked" state, normalized by the chain length.

Inset shows the shear rate dependence of the fraction of time that the chain spends in a locked state over the whole trajectory. For more details and the definitions of the quantities, see text. [Color figure can be viewed in the online issue, which is available at wileyonlinelibrary. com.]

greater than $99.7 \%$ of the chain contour length. Although not shown, the trends are similar if we use $99.9 \%$ of the contour length instead of $99.7 \%$. It is clearly observed that the average residence strain scales linearly with chain length (number of Kuhn steps) at high-shear rates, thereby producing almost universal results for the normalized residence times. The inset shows the dependence on Peclet number of the fraction of the total trajectory over which the chain is "orientationally locked" in the fully stretched state $\left(F_{\text {lock }}\right)$. Note that, for $\mathrm{Pe} \geq 10^{4}$, this fraction is quite close to 1 , which shows the existence of a significant proportion of locked states in the trajectory. Similar to results for $\dot{\gamma} t_{\mathrm{res}} / \mathrm{N}_{\mathrm{K}}$, $F_{\text {lock }}$ is almost independent of chain length at high-shear 


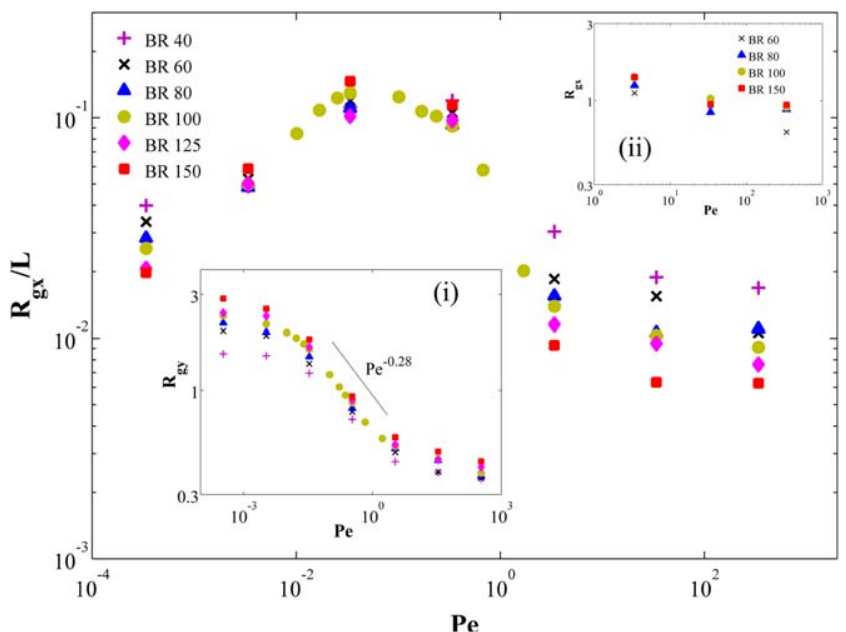

Figure 5. Shear rate dependence of the chain elongation $\boldsymbol{R}_{g x}$ normalized by chain contour length $L$ for BR chains of different length, with hydrodynamic interaction (HI) and no excluded volume (EV).

For all results, the hydrodynamic diameter of the beads is equal to one Kuhn length (represented by one rod in the BR model). Inset (i) shows the shear rate dependence of the coil thickness, represented by $\boldsymbol{R}_{g y}$, for chains of different lengths, when only HI is present. The solid line marks the region of shear rates where $\boldsymbol{R}_{g y}$ follows a power law with an exponent of approximately $-1 / 4$, and inset (ii) shows that the chain forms a coil with $R_{g x}$ of roughly one Kuhn length at high shear rates, for all chain lengths considered. [Color figure can be viewed in the online issue, which is available at wileyonlinelibrary. com.]

rates. Thus, the results in Figure 4(b) explain the chain length independence of coil elongation values (normalized by chain length) at high-shear rates.

\section{Effect of HI (without EV)}

For most simulations of the BR model with HI discussed in what follows, a bead hydrodynamic diameter equal to one Kuhn length is used (corresponding to $\mathrm{h}^{*}=0.5$ ). Figure 5 and inset (i) show the variation of $R_{g x}$ and $R_{g y}$, respectively, with shear rate for this case. The coil elongation (Figure 5) shows an initial increase with shear rate and then a decrease at high-shear rates. Although these trends in both the coil elongation and thickness are qualitatively similar to those obtained without $\mathrm{HI}$ and $\mathrm{EV},{ }^{11}$ the plateau in $R_{g x}$ that constitutes Regime 2 in the earlier studies ${ }^{11,12}$ without $\mathrm{HI}$ is reduced to nothing more than a local maximum in $R_{g x}$ when $\mathrm{HI}$ is present for the chain lengths of up to 150 rods considered in this study. The coil elongation shows a slow decrease after the maximum followed by an abrupt transition to very low values at the highest shear rates. Also, the decrease in $R_{g x}$ depends on chain length and is much steeper (power law exponent about -0.75 for 60 rods and -0.93 for 125 rods) than without $\mathrm{HI}$ or EV (power-law exponent of -0.15 for all chains in Ref. 11). Additionally, the maximum occurs at an $R_{g x} / L$ value of about 0.1 , which is lower than the corresponding value of 0.2 obtained for the plateau without $\mathrm{HI}$ in earlier simulations ${ }^{11,12}$ as well as for the results for EV without HI presented earlier in this article (Figure. 2). These trends are consistent with the simulation results for a chain
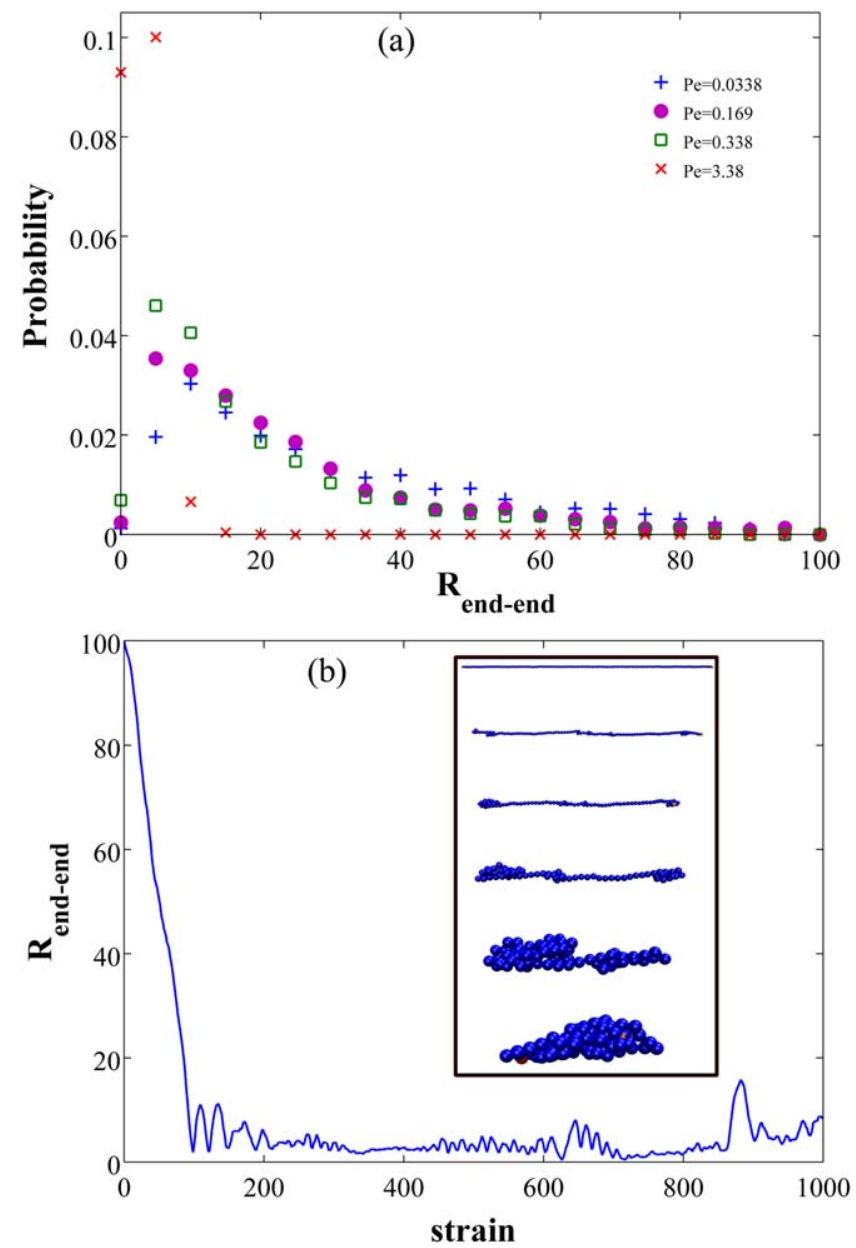

Figure 6. (a) The probability distribution of chain endto-end distance at different shear rates for a BR chain of 100 rods, when only $\mathrm{HI}$ is present between beads with a hydrodynamic diameter of one Kuhn length $\left(h^{*}=0.5\right)$, and $(b)$ the time variation of the end-to-end distance of a BR chain of 100 rods from a stretched state, with only $\mathrm{HI}$ present, at a shear rate with $\mathrm{Pe}=3.38(\dot{\gamma}=100)$.

Inset shows the corresponding sequence of chain configurations to form a coiled state starting from a stretched state, where time increases from top to bottom. Note that the chain shows negligible stretching once a coiled state is attained. [Color figure can be viewed in the online issue, which is available at wileyonlinelibrary. com.]

of 100 rods with $\mathrm{HI}$ in the earlier study of Sendner and Netz. ${ }^{9}$

In addition to the lower maximum value of $R_{g x} / L$, another significant difference from chains without $\mathrm{HI}$ is that the value of $R_{g x}$ at high-shear rates in Regime 3, shown in the inset (ii) to Figure 5, is very small, only around one Kuhn length (which is the length of one rod for the BR model). Hence the ratio $R_{g x} / L$ monotonically decreases with chain length at high-shear rates in Figure 5. The variation of the coil thickness with shear rate (inset (i)) is similar to those observed earlier, ${ }^{11,12}$ including the appearance of all three regimes. In Regime $1, R_{g y}$ remains close to the equilibrium (no-flow) value; in Regime 2, where $R_{g x}$ goes through a maximum, $R_{g y}$ decreases with a power law exponent of 


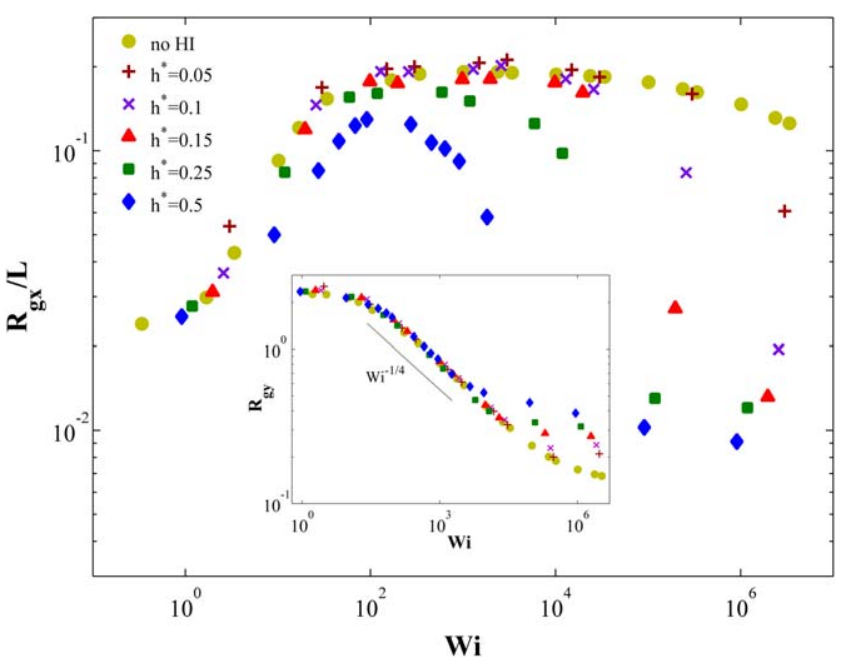

Figure 7. Weissenberg number dependence of the chain elongation for varying levels of $\mathrm{HI}$, modeled by different values of the bead hydrodynamic diameter (controlled by the parameter $h^{*}$ in the simulations) for a BR chain of 100 rods.

The inset shows the Weissenberg number dependence of the coil thickness $\left(R_{g y}\right)$ for varying levels of HI. The solid line marks the region of shear rates where $R_{g y}$ follows a power law with an exponent of approximately $-1 / 4$. [Color figure can be viewed in the online issue, which is available at wileyonlinelibrary.com.]

about $-1 / 4$; and in Regime $3, R_{g y}$ saturates at very highshear rates. Furthermore, the values of $R_{g y}$ become independent of chain length for longer chains at high shear rates, which is consistent with the earlier observations. ${ }^{11,12}$

The absence of a plateau in chain stretch and the greater tendency of the BR chain to form a coil are also highlighted in Figure 6a. This shows the probability distribution of the chain end-to-end distance for a BR chain of 100 Kuhn steps at different shear rates. Note that at all shear rates the probability distribution shows a maximum at a low value of endto-end distance, with the highly stretched states being significantly less probable. Also, the probability distribution shows a sudden transition between the shear rates of $\dot{\gamma}=10$ $(\mathrm{Pe}=0.338)$ to $\dot{\gamma}=100 \quad(\mathrm{Pe}=3.38)$, corresponding to the abrupt transition to very low values of $R_{g x}$ already shown in Figure 5. The absence of the largely uniform probability distribution of coil elongation observed for the shear rates in Regime 2 in the absence of $\mathrm{EV}$ and $\mathrm{HI}^{7,11}$ further confirms that HI promotes the clustering of beads and thus significantly reduces the probability of stretched states.

This clustering due to $\mathrm{HI}$ at high-shear rates with the BR model in Regime 3 is highlighted further in the inset of Figure $6 \mathrm{~b}$, which shows the coiling of a BR chain of 100 rods, starting from an initial fully stretched state, at a shear rate of $\dot{\gamma}=100 \quad(\mathrm{Pe}=3.38)$. This inset presents the sequence of chain configurations leading to a coiled state, where time increases from top to bottom and the snapshots are zoomed in at higher times to show the clustering of beads more clearly. Figure $6 \mathrm{~b}$ shows the corresponding temporal variation of the end-to-end distance, which shows a sharp decrease to a coiled state with no further stretching. This behavior of tumbling in the coiled state with HI contrasts with the behavior in Regime 3 in earlier simulations that omitted HI (discussed in Ref. 11), in which the chain tumbles by a sequence of stretched and coiled states, with the maximum stretch decreasing with increasing shear rate, but not to the extent seen when HI is present. We will show later that this extreme clustering and overlapping of beads is physically unrealistic, and disappears once bead-bead excluded volume interactions are introduced or the chain model is discretized further.

Figure 7 and its inset show the effect of bead hydrodynamic diameter on the variation of the coil elongation and thickness with shear rate, respectively, for a chain of 100 rods in the absence of any excluded volume interactions. As discussed earlier, the hydrodynamic diameter is controlled by the parameter $h^{*}$, where $h^{*}=0.5$ represents a hydrodynamic diameter of one rod length (or one Kuhn step for the BR model). The trends in Figure 7 are presented as functions of Weissenberg number $\mathrm{Wi}$, rather than Peclet number to indicate the similarity of the results (especially for the coil thickness) at low and intermediate shear rates. Results in Figure 7 show that, at any $\mathrm{Wi}, R_{g x}$ decreases with increasing bead hydrodynamic diameter, especially at high Wi, indicating an increased clustering of beads at higher HI. As expected, for low bead hydrodynamic diameter, $\mathrm{h}^{*}=0.05$, the elongation approaches that for a 100-rod chain without HI over a wide range of shear rates, although the transition to Regime 3 occurs at a somewhat lower value of $\mathrm{Wi}$ for $\mathrm{h}^{*}=0.05$ than it does for $\mathrm{h}^{*}=0$. As $\mathrm{h}^{*}$ increases, this transition becomes more abrupt and occurs at ever lower values of Wi. Also, the value of the maximum coil elongation decreases with increasing $h^{*}$, and the range of shear rates over which there is a plateau in elongation also decreases systematically. Interestingly, even in Regime 1 the chain deformation eventually decreases when $\mathrm{h}^{*}$ approaches 0.5 . The inset presents the dependence of $R_{g y}$ on Wi for different values of $\mathrm{h}^{*}$, which shows systematic deviations from the results with no HI only at high-shear rates. Similar to the results for $R_{g x}$, the saturation in $R_{g y}$ at high flow rates occurs at lower $\mathrm{Wi}$, and hence at higher values of $R_{g y}$, when $\mathrm{h}^{*}$ increases, highlighting an early transition to Regime 3 . At lower shear rates, all the results are almost identical with each other.

\section{Effects of HI, EV and bead-bead attraction (EV-theta)}

Figure 8 and its inset present the chain deformations along the flow and shear-gradient directions when both hydrodynamic interaction (HI) and bead-bead excluded volume interactions are included in the BR model. For all these simulations, the bead hydrodynamic diameter is taken as one Kuhn length, and so $\mathrm{h}^{*}=0.5$. Figure 8 presents the Peclet number variation of $R_{g x} / L$ for chains of different lengths using EV and EV-theta interactions. Similar to the case of a chain with HI, chains longer than 150 rods are not considered owing to high-computational cost. It is clear from Figure 8 that in the moderate and high-shear regions the results for EV and EV-theta chains are qualitatively similar. The overall trends agree well with those obtained for chains where only EV acts between beads (Figure 2), with an increase in coil elongation followed by a plateau-like region (Regime 2) and a slow increase at extremely high shear rates (Regime 3). However, the value of $R_{g x} / L$ at the plateau is about 0.2 for $\mathrm{EV}$, which is close to that obtained for chains where neither HI nor any bead-bead LJ interactions are present, compared to a value of roughly 0.1 for EV-theta chains, 


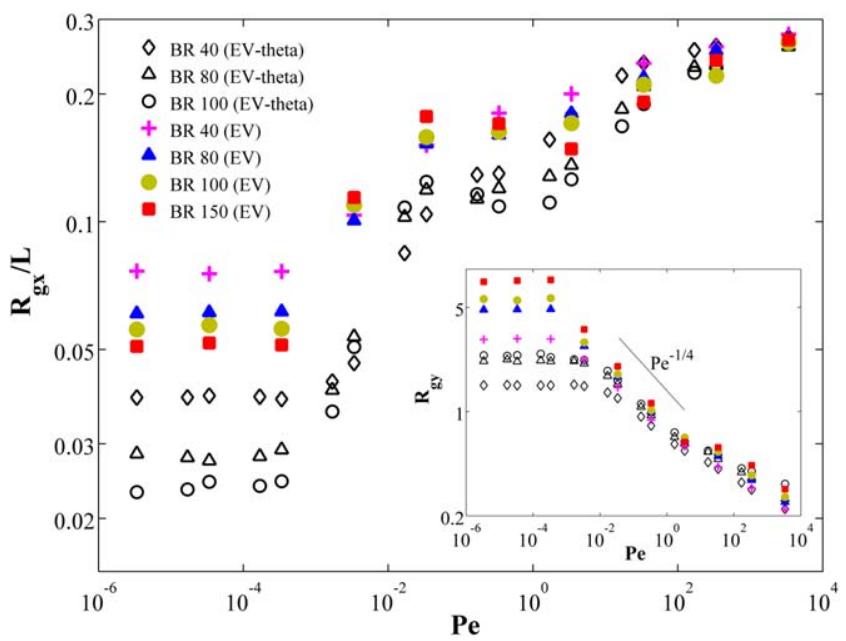

Figure 8. Shear rate dependence of the coil elongation normalized by chain length for chains of different length, when both EV and $\mathrm{HI}$ are present.

$\mathrm{EV}$ is modeled in two ways-by the repulsive part of the $\mathrm{LJ}$ potential with $\sigma^{*}=1$ (denoted by "EV-theta"), and by the full $L J$ potential with the value of $\varepsilon^{*}$ tuned such that the size of the chain at equilibrium is similar to that of a theta coil (denoted by "EV-theta"). The bead hydrodynamic diameter is taken as one Kuhn length for all cases. The inset shows the dependence on Peclet number of the coil thickness $R_{g y}$ for chains of different length, when both EV and HI are active between beads. The solid line marks the region of Peclet numbers where $R_{g y}$ shows a power law with an exponent of approximately $-1 / 4$. [Color figure can be viewed in the online issue, which is available at wileyonlinelibrary.com.]

which is similar to the corresponding value for chains where only HI acts between beads, as discussed earlier. The shortrange repulsive interaction between beads presumably reduces the clustering of beads mentioned earlier and increases the coil elongation at intermediate shear rates where the plateau occurs. For EV-theta chains, additional long range attractive interactions between beads counterbalances the effect of short range repulsion and reduces the elongation to a value similar to that of a chain without $\mathrm{EV}$ in Regimes 1 and 2. However, in Regime 3, the values of $R_{g x} / L$ show a slow increase at large shear rates, similar to the case of chains with only EV discussed earlier in this article, and in contrast to the results obtained with HI only. As mentioned earlier for chains with only EV, this increase is caused by increased locking in the fully stretched state, similar to what is shown in the inset of Figure 3. In accordance with the results for coil elongation, the results for $R_{g y}$ for $\mathrm{EV}$ and EV-theta shown in the inset of Figure 8 are quite similar to those observed in the earlier case with only EV. Again, there is a low shear rate region of nearly constant $R_{g y}$ (Regime 1), followed by a power-law decrease (exponent around $-1 / 4$ for the chains considered) at intermediate shear rates (Regime 2). At extremely high shear rates in Regime 3, increasing orientational locking causes a further decrease of $R_{g y}$, but with a reduced slope compared to Regime 2 .

\section{Comparison of effects of different mechanisms}

Figures $9 \mathrm{a}$ and $\mathrm{b}$ compare the coil elongation and tumbling behaviors for a chain of 100 rods (using the BR model) with increasing shear rates, for different combinations of mecha-
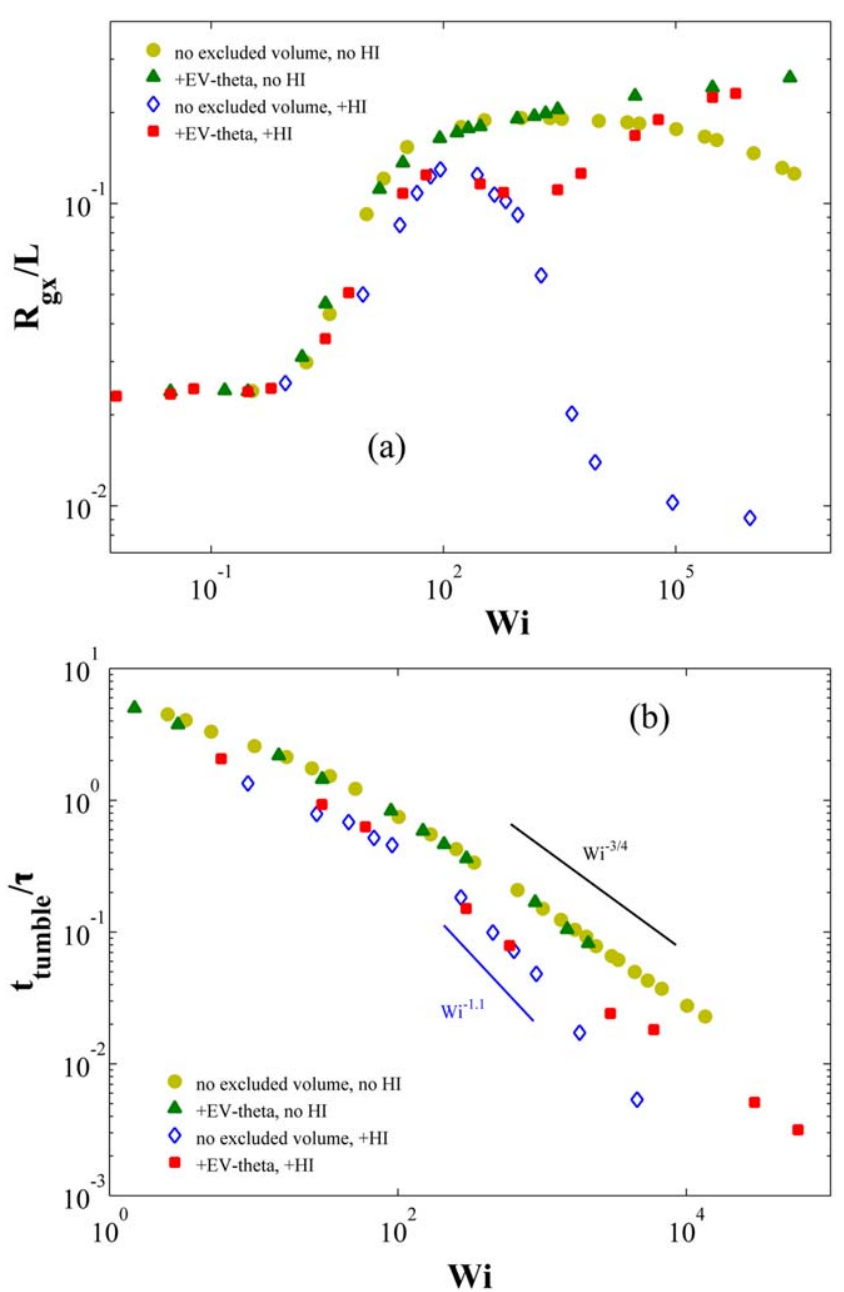

Figure 9. Shear rate dependence of (a) the chain elongation, and (b) the tumbling time (normalized by the end-to-end relaxation time of the chain) for a BR chain of 100 rods, when EVtheta and $\mathrm{HI}$ are present or absent.

The "+ " sign and "no" indicate the mechanism that is present and not present, respectively. For all results shown here, "EV-theta" means that the full $\mathrm{LJ}$ potential is used for bead-bead interactions with $\sigma^{*}=1$ and the value of $\varepsilon^{*}$ tuned such that the coil size at zero shear rate is same as that of a theta coil without excluded volume interactions (for this case, $\varepsilon^{*}=0.44$ ). The bead hydrodynamic diameter is taken as one Kuhn length for all cases here. The solid lines indicate the different power laws shown in the different cases. [Color figure can be viewed in the online issue, which is available at wileyonlinelibrary.com.]

nisms. The results are plotted against Weissenberg number, instead of the Peclet number, to highlight the similarity in the results at low shear rates. In all cases, the bead-bead excluded volume interaction is modeled by the EV-theta potential with $\sigma^{*}=1$, whereas the bead hydrodynamic diameter is equal to the length of a single rod (which represents one Kuhn step in the BR model). Figure 9 a shows that $R_{g x} / L$ with only EV-theta and no HI (green symbols) approximately agrees with results in the absence of both EV and HI, (yellow symbols) except at extremely high shear rates of Regime 3 , where the LJ potential between the beads hinders the bead overlap that causes chain shrinkage. However, at intermediate shear rates (in Regime 2), $R_{g x} / L$ shows a gradual increase with shear rate even with the 
EV-theta potential, instead of the flat plateau obtained for chains in the absence of $\mathrm{HI}$ and $\mathrm{EV}$. The presence of $\mathrm{HI}$ $\left(\mathrm{h}^{*}=0.5\right.$ in this case), with or without EV-theta, reduces the chain deformation in Regime 1 and lowers the maximum $R_{g x} /$ $L$ to about 0.1 (compared to 0.2 without EV-theta or HI). Without bead-bead interactions (blue symbols), the coil elongation shows a very short plateau followed by a sharp transition to chain shrinkage, because HI without excluded volume interactions promotes bead clustering, as discussed earlier. With EVtheta and HI (red symbols), the elongation decreases below the maximum and then increases again and eventually merges with the results obtained with EV-theta only at extremely high shear rates, instead of showing chain shrinkage in Regime 3, presumably due to orientational locking in the extended state at high shear rates, as discussed earlier. Thus, the "theta" condition obtained by dropping the bead-bead interaction potential gives similar results to that obtained by balancing a long-range attraction with a short-range repulsion except at high shear rates where the presence of the short-range repulsion prevents coil collapse.

The variation of the chain tumbling times (normalized by the end-to-end relaxation time $\tau$ ) with $\mathrm{Wi}$ in Regimes 1 and 2 is shown in Figure 9b. The tumbling time is the average time needed for an extended chain to rotate through the flow direction, coil-up, and then re-extend, as discussed elsewhere ${ }^{7}$ and calculated using the algorithm explained in Ref. 12. At highshear rates, the coiling-up process occurs by means of growth of chain loops formed at chain ends, also discussed in Ref. 12. Average chain loop sizes (which set the value of $R_{g y}$ ) in this range of shear rates are similar to those reported earlier (see Ref. 12 for a comparison of the values of $R_{g y}$ ). The trends for the tumbling time are consistent with those expected based on the observations for the coil elongation in Figure 9a. The results for cases without $\mathrm{HI}$, with or without EV-theta, agree with one another and show a power law regime with an exponent of about $-3 / 4$ in the range of shear rates where the plateau is observed in $R_{g x}$ (Regime 2), which is explained in Ref. 12. When both HI and EV-theta are present, the tumbling times are reduced below those in the absence of HI, but a similar scaling law is observed, consistent with the fact that the coil elongation roughly follows a plateau in Regime 2 . The lower values of the observed tumbling time are expected due to reduced maximum elongation in this case. When only $\mathrm{HI}$ is present, and no EV-theta, the tumbling time decreases with a steeper exponent of about -1.1 . The steep decrease of the tumbling time with shear rate for chains with HI, but no interaction between the beads is consistent with the tendency of the beads to cluster and overlap with each other. The fast tumbling gives the chains little time to stretch in the flow direction before they are rotated into an orientation that causes compression. At the same time, the condensed state of the coil gives it a small aspect ratio, like a sphere, which tends to rotate at a rate proportional to the imposed shear rate. In fact, it is wellknown that a liquid droplet that is more viscous than the surrounding fluid tumbles, but stretches very little in a shear flow, no matter how high the shear rate. ${ }^{13}$ Thus, the increased rate of tumbling and the decreased stretch reinforce each other, leading toward coil collapse, unless there is bead-bead repulsion to keep the beads from clustering densely.

\section{Effects of chain resolution}

The results in Ref. 12, in the absence of HI and any excluded volume interactions, have shown that the chain response at

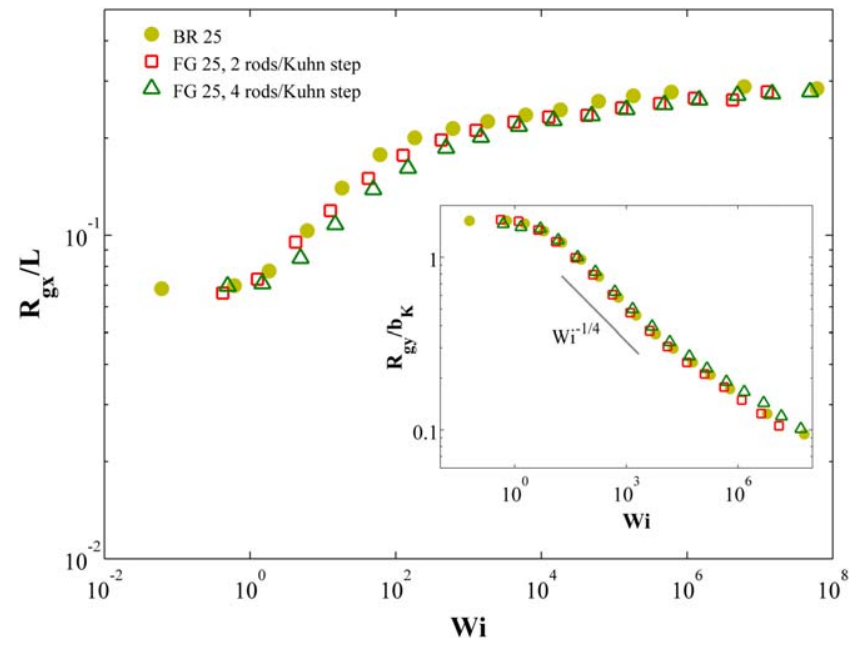

Figure 10. Variation of the coil elongation (normalized by the contour length $L$ ) with shear rate (represented by Weissenberg number) for a chain that consists of 25 Kuhn steps, at different levels of resolution, using the BR and FG models, including EV (no $\mathrm{HI}$ ).

Inset shows the dependence of the coil thickness, normalized by the Kuhn length, on shear rates for the same cases. The solid line indicates the region of shear rates where $\boldsymbol{R}_{g y}$ approximately follows a power law with an exponent of $-1 / 4$. The legend indicates the type of model used (BR or FG) followed by the number of Kuhn steps (25), and then the number of rods that constitute one Kuhn step. For all models used, $\varepsilon^{*}$ is 1.0 and $\sigma^{*}$ is varied so that the coil sizes are similar in the absence of shear flow $\left(\sigma^{*}=0.6\right.$ for the BR chain). [Color figure can be viewed in the online issue, which is available at wileyonlinelibrary.com.]

ultra-high shear rates in Regime 3 is highly sensitive to the chain resolution, with the onset of the decrease in the coil elongation being pushed to higher shear rates with increasing levels of discretization made possible by use of a fine-grained (FG) model that resolved the chain below the level of a Kuhn step. Here, we use the FG model in the presence of EV and HI to study the response of the chain over a wide range of shear rates, when the chain resolution is increased systematically. The studies presented here with EV (and without $\mathrm{HI}$ ) and with $\mathrm{HI}$ (in the absence of EV) are performed on chains consisting of 25 and 12 Kuhn steps, respectively, at varying degrees of resolution. The values of the bending stiffness needed to attain a certain number of rods per Kuhn step in the FG chain (i.e., the chain resolution) are provided in Ref. 12.

Figure 10 shows the variation of the coil elongation (normalized by L) with the Weissenberg number, for BR and FG chains of different resolutions, all consisting of a total of 25 Kuhn steps with no HI but with EV. For all resolutions, we used $\varepsilon^{*}=1.0$, while tuning the values of $\sigma^{*}$ at each level of resolution such that the coil sizes are similar in the absence of shear flow (Note that $\sigma^{*}=0.6$ for the BR model for these simulations. Since there are more beads, and hence more pairwise interactions, in chains with higher levels of resolution, the same values of $\varepsilon^{*}$ and $\sigma^{*}$ will give a larger coil size at equilibrium with increasing resolution.). Interestingly, although minor differences exist between the results at relatively low shear rates, the behavior at high-shear rates is independent of the level of chain discretization, in sharp contrast to the results in Ref. 12 for cases without $\mathrm{HI}$ and 


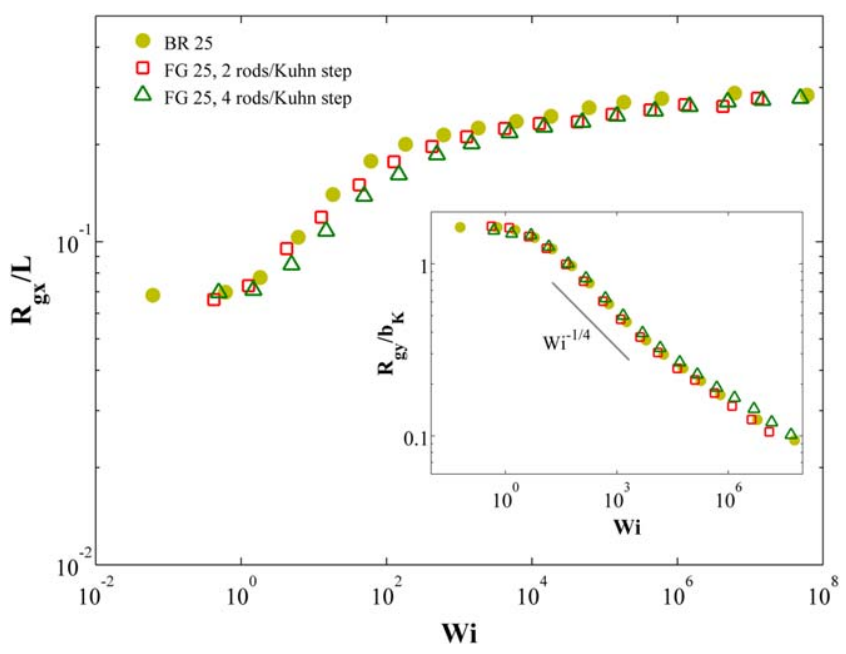

Figure 11. Variation of the coil elongation (normalized by the contour length L) with shear rate (represented by Weissenberg number) for a chain of 12 Kuhn steps, at different levels of resolution, using the $B R$ and FG models, including $\mathrm{HI}$ (without any bead-bead excluded volume interactions).

Inset shows the dependence of the coil thickness, normalized by the Kuhn length, on shear rate for the same cases. The solid line indicates the region of shear rates where $\boldsymbol{R}_{g y}$ approximately follows a power law with an exponent of $-1 / 4$. The meaning of the legend is the same as in Figure 10. For all models used, $h^{*}$ is fixed at 0.5. [Color figure can be viewed in the online issue, which is available at wileyonlinelibrary.com.]

excluded volume interactions. The variation of the coil thickness with Weissenberg number (shown in the inset) is also similarly consistent over varying degrees of chain resolution. These results indicate that our observations presented earlier for BR chains with EV (and similar ones shown for a chain of 100 rods in Ref. 9) will remain valid over the entire range of shear rates considered in this study.

Figure 11 presents a similar study in the presence of $\mathrm{HI}$ (without excluded volume interactions), for chains of varying resolutions that consist of 12 Kuhn steps. For all levels of resolution used in this case, we use $\mathrm{h}^{*}=0.5$. The behavior of $R_{g x} / L$ for different model discretizations is similar to those presented in Ref. 12, with the plateau in coil elongation becoming more prominent with increasing chain resolution, consequently pushing the onset for the decrease in chain stretch to higher Wi. A similar behavior is observed for the coil thickness as well (given in the inset), with the onset of saturation in $R_{g y}$ occurring at larger Weissenberg numbers with increasing chain resolution. These results imply that our observations regarding the clustering of beads in the presence of $\mathrm{HI}$, leading to highly compact configurations, is most likely an artifact due to insufficient chain resolution. In the continuous bending limit (i.e., when there are an infinite number of rods to model one Kuhn step), when only HI is acting (without any excluded volume interactions), the onset of the decrease might be pushed to $\mathrm{Wi} \rightarrow \infty$ and only Regimes 1 and 2 will be present, similar to the observations in Ref. 12.

\section{Performance of the CG model}

Since most of the earlier studies have used bead-spring chains (i.e., CG chains) for prediction of polymer chain

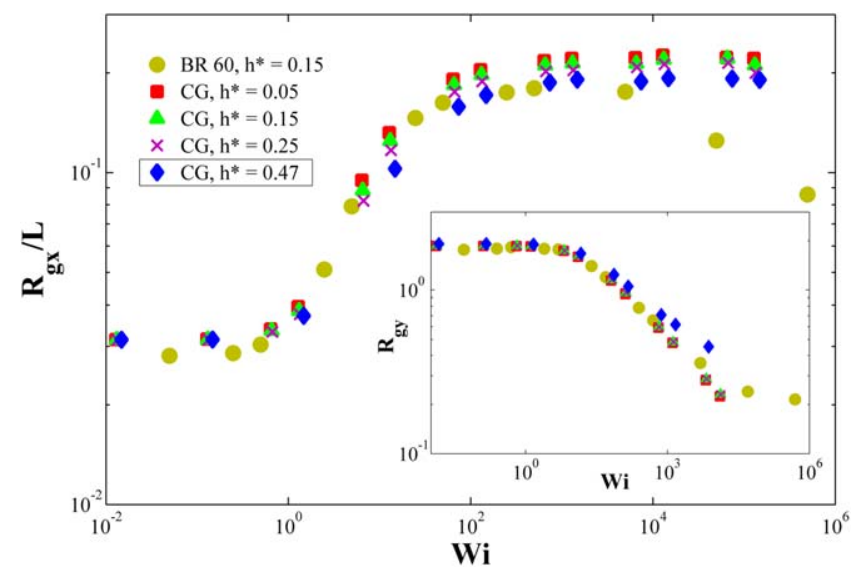

Figure 12. Variation of the coil stretch (normalized with the chain contour length) with shear rate (represented by Wi) for BR and CG models for a chain of 60 Kuhn steps, in the presence of HI.

Note that most simulations are performed with the CG model with $v=10$ (6 springs) for different values of $h^{*}$, while only one simulation uses the BR model with $h^{*}=0.15$. The estimated "equivalent" $h$ " for the CG model with $v=10$ is highlighted by a box (details given in text). The inset shows the results for the coil thickness. [Color figure can be viewed in the online issue, which is available at wileyonlinelibrary.com.]

dynamics in flow, it becomes important to know if the predictions of the $\mathrm{CG}$ and the BR models agree well in the presence of $\mathrm{HI}$ (note that a comparison of the predictions of the $\mathrm{CG}$ and $\mathrm{BR}$ models in the absence of $\mathrm{HI}$ is provided in Ref. 11). Figures 12 and 13 compares the predictions of the $\mathrm{BR}$ and $\mathrm{CG}$ model in the presence of HI. For this study, the BR model for a chain of 60 Kuhn steps with $\mathrm{h}^{*}=0.15$ is used. Two different resolutions are used for the CG - one with $v=10$ (used in the results of Figure 12) and another with $v=6$ (used in the results of Figure 13). Note that $v$ denotes the number of Kuhn steps mimicked by each spring, as mentioned earlier. For the CG model with $v=10$ (6 springs), the equilibrium separation between drag centers is $\sqrt{10} \approx 3.16$ (where the unit of length is one Kuhn step). Thus, to mimic the BR chain with $\mathrm{h}^{*}=0.15$, using a chain with 6 springs, the "equivalent" value of $\mathrm{h}^{*}$ should be $\mathrm{h}^{*}=3.16^{*} 0.15=0.47$, so that as the number of drag centers is reduced, the strength of HI per drag center is correspondingly increased (an alternative interpretation of the same is obtained by equating the strength of hydrodynamic coupling, $\mathrm{N}_{\text {beads }}{ }^{1 / 2} \mathrm{~h}^{*}$, across models of different resolutions, ${ }^{8}$ where $\mathrm{N}_{\text {beads }}$ is the total number of beads in the chain). Similarly, for the CG model with $v=6$ (10 springs), a similar calculation gives an equivalent value of $h^{*}=0.37$. However, note that this calculation is based on the equilibrium spring length. Unlike the BR model where the distance between drag centers is fixed, the separation between beads undergoes significant fluctuations in a CG model.

Figures 12 and 13 compare the coil stretch predicted by the BR model and the CG model with $v=10$ and $v=6$, respectively, with the corresponding values of the coil thickness shown in the insets. All CG calculations are done with different levels of HI to show its effect on the predictions, although only one value of $\mathrm{h}^{*}$ (the one highlighted by a green box) mimics the level of $\mathrm{HI}$ in the BR model. Note 


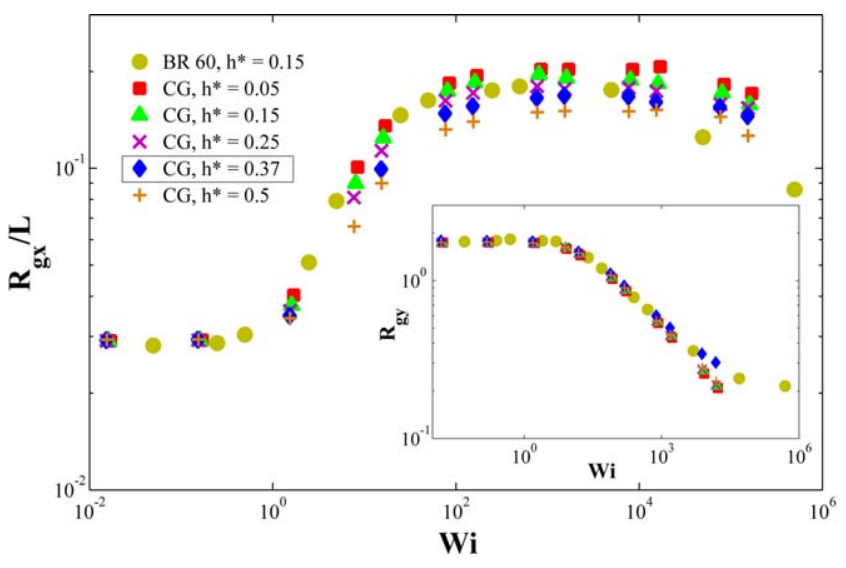

Figure 13. The same as Figure 12, except that the CG model used for most of the simulations uses $v=6$ (10 springs) for different values of $\mathbf{h}^{*}$, while only one simulation uses the BR model with $\mathrm{h}^{\star}=0.15$.

The estimated "equivalent" $h$ " for the CG model with $v=6$ is highlighted by a box (details given in text). The inset shows the results for the coil thickness. [Color figure can be viewed in the online issue, which is available at wileyonlinelibrary.com.]

that, similar to the BR model with different levels of HI, $R_{g x}$ in the CG model is insensitive to $\mathrm{HI}$ at low and intermediate shear rates, but the plateau value in Regime 2 decreases slightly with increasing $\mathrm{h}^{*}$. Interestingly, the decrease in $R_{g x}$ in Regime 3 is delayed in the CG model, relative to the BR model (However, it was shown earlier that the onset of chain compression in Regime 3 for the BR model in the presence of $\mathrm{HI}$ (and absence of EV) is pushed to arbitrarily high shear rates with increasing chain resolution, beyond one Kuhn step, for the FG model). Note in Figure 12 that for a beadspring chain with six springs, the value of $h^{*}$ that yields a plateau value of $R_{g x}$ matching the value for the bead-rod model is about 0.47 , which agrees with the estimated "equivalent" value calculated earlier. However, for the 10spring CG model, the value of $\mathrm{h}^{*}$ that yields the best match to the plateau in $R_{g x}$ for the bead-rod model appears to be about 0.25 , instead of the estimated equivalent value of 0.37 , although the results are pretty close (see Figure 13). This can be attributed to the loss in accuracy of the spring law when the number of Kuhn steps per spring is small (6 in this case). Note that, for the Cohen-Padé approximation, the coil size at equilibrium is slightly underpredicted for $v=6$ (For this case of a chain of 60 Kuhn steps, $R_{g}$ is roughly 3.0 instead of 3.16 for a Gaussian coil. Also, in the absence of $\mathrm{HI}$, we showed in our earlier work ${ }^{11}$ that for a CG model of 83 springs with $v=6$, the mean end-to-end distance is approximately 19.6 instead of the theoretical value of 22.3, thus, again indicating that the CG model is not entirely accurate when $v=6$.). The largest value of $\mathrm{h}^{*}$ that can reasonably be used in bead-spring simulations is around 0.5 , since larger values than this imply frequent overlap in hydrodynamic volume, and the RPY tensor becomes grossly inaccurate. Thus, unless the HI parameter $h^{*}$ appropriate for the BR model is much smaller than 0.5 , there will be a serious limit to the degree of coarse graining that is realistically possible with the bead-spring model, while still capturing the hydrodynamic interactions realistically.

\section{Summary}

We have presented a detailed study of the effects of hydrodynamic interaction $(\mathrm{HI})$ and excluded volume $(\mathrm{EV})$ on the deformation of polymer chains subjected to a wide range of shear rates. For most investigations presented, we have adopted a bead-rod (BR) chain, where each rod is modeled by a stiff, nearly inflexible Fraenkel spring, which mimics a single Kuhn step in the chain. The EV and HI between beads are computed using the Lennard-Jones (LJ) potential and RotnePrager-Yamakawa (RPY) tensor, respectively. Our results show three distinct regimes in most cases, just as the results without $\mathrm{HI}$ and excluded volume interactions given in the earlier studies. ${ }^{11,12}$ These regimes can be identified by plots of polymer deformation as a function of the shear rate made dimensionless with either the relaxation time of the whole chain, which gives the Weissenberg number $\mathrm{Wi}$, or with the relaxation time of a single rod, which gives the rod Peclet number Pe. With increasing shear rate, these regimes are as follows. Regime 1: a low shear-rate regime with Wi less than around 100 or so (depending on chain length) in which the coil elongation in the flow direction $R_{g x}$ increases with shear rate while the coil thickness in the gradient direction $R_{g y}$ remains nearly constant. Regime 2: a modest shear rate regime with $\mathrm{Wi}>100$ or so and $\mathrm{Pe}<1$, having a plateau in $R_{g x}$ and a $-1 / 4$ power-law decrease in $R_{g y}$ with $R_{g y}$ independent of chain length. Regime 3: an ultra-high shear rate regime with $\mathrm{Pe}>1$, where the shear is strong enough to highly orient individual rods and the results are sensitive to the modeling details.

While excluded volume expands the coil size in Regime 1, it has little effect on coil dimensions in Regime 2. However, in the presence of excluded volume, the plateau observed in earlier results ${ }^{1,12}$ is replaced by a weakly increasing coil elongation with shear rate. An increase in HI (increase in the bead hydrodynamic diameter) systematically reduces the chain elongation in Regimes 1 and 2, to such an extent that the maximum elongation in Regime 2 is lowered by a factor of roughly two when the bead hydrodynamic diameter equals the length of each rod (or Kuhn step), relative to chains without $\mathrm{HI}$ and $\mathrm{EV}$.

In Regime 3, if there is no bead-bead repulsion, the BR chain eventually suffers a decrease in elongation with increasing shear rate even with no $\mathrm{HI}$ or excluded volume interactions, and HI strongly exaggerates this tendency, so that the coil elongation $R_{g x}$ even drops below the value it has in the absence of flow, down to a length of roughly a single rod length. This coiled-up state in the presence of $\mathrm{HI}$ is permitted by the overlap of beads, and even small levels of short-range bead-bead repulsion suppress this coil collapse. This collapse is removed even if the short-range bead repulsion is accompanied by longer-range attraction, such that the coil achieves a theta condition at equilibrium. In the presence of bead-bead excluded volume interactions, Regime 3 is characterized by increasing levels of orientational "locking" in the fully extended state. Here "locking" refers to periods in which the chain remains completely extended with all rods aligned in the flow direction. Our simulations with a fine-grained FG model, which is resolved to length scales lower than one Kuhn step, highlights the universality of the chain deformation behavior at high shear rates in the presence of excluded volume interactions. Similar simulations in the presence of HI (without any excluded volume interactions) show that the chain compression at high shear rates obtained with the BR model is most likely a simulation artifact, (or at least is very sensitive to simulation details) and the onset of such a decrease in chain 
stretch will be pushed to arbitrarily high shear rates with either inclusion of short-range bead-bead repulsion or with increasing model resolution attained by inclusion of a bending potential. Simulations with the coarse-grained (CG) bead-spring model with HI give results similar to those of the BR model, when the value of the HI parameter is selected appropriately to give a similar overall strength of $\mathrm{HI}$ in both $\mathrm{CG}$ and $\mathrm{BR}$ models.

\section{Acknowledgment}

We gratefully acknowledge the support of the National Science Foundation, under grant DMR 0906587. Any opinions, findings, and conclusions or recommendations expressed in this material are those of the authors and do not necessarily reflect the views of the National Science Foundation (NSF).

\section{Literature Cited}

1. Bird R, Curtiss B, Armstrong CF, Robert C, Hassager O. Dynamics of Polymeric Liquids. Kinetic Theory. New York: Wiley-Interscience; 1987.

2. Smith DE, Babcock HP, Chu S. Single-polymer dynamics in steady shear flow. Science. 1999;283:1724-1727.

3. Hur JS, Shaqfeh ESG, Babcock HP, Smith DE, Chu S. Dynamics of dilute and semidilute DNA solutions in the start-up of shear flow. $J$ Rheol. 2001;45:421-450.
4. Schroeder CM, Teixeira RE, Shaqfeh ESG, Chu S. Characteristic periodic motion of polymers in shear flow. Phys Rev Lett. 2005;95: 018301.

5. Doyle PS, Shaqfeh ESG, Gast AP. Dynamic simulation of freely draining flexible polymers in steady linear flows. J Fluid Mech. 1997;334:251-291.

6. Petera D, Muthukumar M. Brownian dynamics simulation of beadrod chains under shear with hydrodynamic interaction. J Chem Phys. 1999;111:7614-7623.

7. Hur JS, Shaqfeh ESG, Larson RG. Brownian dynamics simulations of single DNA molecules in shear flow. $J$ Rheol. 2000;44:713-742.

8. Hsieh, Chih-Chen, Li, Lei, Larson, Ronald G. Modeling hydrodynamic interaction in Brownian dynamics: simulations of extensional flows of dilute solutions of DNA and polystyrene. J Non-Newtonian Fluid Mech. 2003;113:147-191.

9. Sendner C, Netz RR. Single flexible and semiflexible polymers at high shear: Non-monotonic and non-universal stretching response. Eur Phys J E. 2009;30:75-81.

10. Wolfgang P, Binder K, Heermann DW, Kremer K. Dynamics of polymer solutions and melts. Reptation predictions and scaling of relaxation times. $J$ Chem Phys. 1991;95:7726.

11. Dalal IS, Hoda N, Larson RG. Multiple regimes of deformation in shearing flow of isolated polymers. $J$ Rheol. 2012;56:305.

12. Dalal IS, Albaugh A, Hoda N, Larson RG. Tumbling and Deformation of Isolated Polymer Chains in Shearing Flow. Macromolecules. 2012;45:9493-9499.

13. Larson RG. The Structure and Rheology of Complex Fluids. New York: Oxford University Press; 1998.

Manuscript received Sept. 18, 2013, and revision received Nov. 29, 2013. 